\title{
A NOTE ON PRECONDITIONING NON-SYMMETRIC MATRICES
}

\author{
ILSE C. F. IPSEN*
}

Abstract. The preconditioners for indefinite matrices of KKT form in [M.F. Murphy, G.H. Golub, and A.J. Wathen: A Note on Preconditioning for Indefinite Systems, SIAM J. Sci. Comput., vol. 21, no. 6, pp 1969-1972 (2000)] are extended to general non-symmetric matrices.

Key words. preconditioner, minimal polynomial

AMS subject classification. $65 \mathrm{~F} 10,15 \mathrm{~A} 23$

In [2] preconditioners for real indefinite matrices of KKT form

$$
\mathcal{A} \equiv\left(\begin{array}{cc}
A & B^{*} \\
C & 0
\end{array}\right)
$$

are presented ${ }^{1}$. The preconditioners $\mathcal{P}$ are of the following form

$$
\left(\begin{array}{cc}
A & B^{*} \\
0 & \pm C A^{-1} B^{*}
\end{array}\right), \quad\left(\begin{array}{cc}
A & B^{*} \\
C & 2 C A^{-1} B^{*}
\end{array}\right), \quad\left(\begin{array}{cc}
A & 0 \\
0 & C A^{-1} B^{*}
\end{array}\right) .
$$

The preconditioned matrices $\mathcal{P}^{-1} \mathcal{A}$ have minimal polynomials of degree at most 4 . Hence a Krylov subspace method like GMRES applied to a preconditioned linear system with coefficient matrix $\mathcal{P}^{-1} \mathcal{A}$ converges in 4 iterations or less, in exact arithmetic.

We extend the preconditioners $\mathcal{P}$ in [2] to general matrices $\mathcal{A}$, by deriving them from LU decompositions of $\mathcal{A}$. As before, the preconditioned matrices $\mathcal{P}^{-1} \mathcal{A}$ and $\mathcal{A} \mathcal{P}^{-1}$ have minimal polynomials of degree at most four.

Let

$$
\mathcal{A} \equiv\left(\begin{array}{cc}
A & B^{*} \\
C & D
\end{array}\right)
$$

be a complex, non-singular matrix where the leading principal submatrix $A$ is nonsingular. Let $S \equiv D-C A^{-1} B^{*}$ be the Schur complement with respect to $A$. Since $\mathcal{A}$ is non-singular, so is $S$. The idea is to factor $\mathcal{A}=\mathcal{L} \mathcal{D} \mathcal{U}$ such that the preconditioned matrix $\mathcal{L}^{-1} \mathcal{A U}^{-1}=\mathcal{D}$ has a minimal polynomial of small degree.

Proposition 1 (Extension of Remark 2 in [2]). If

$$
\mathcal{P} \equiv\left(\begin{array}{cc}
A & B^{*} \\
0 & S
\end{array}\right)
$$

the $n^{2}$

$$
\mathcal{A} \mathcal{P}^{-1}=\left(\begin{array}{cc}
I & 0 \\
C A^{-1} & I
\end{array}\right)
$$

and $\mathcal{P}^{-1} \mathcal{A}$ and $\mathcal{A} \mathcal{P}^{-1}$ have the minimal polynomial $(\lambda-1)^{2}$.

\footnotetext{
* Center for Research In Scientific Computation, Department of Mathematics, North Carolina State University, P. O. Box 8205, Raleigh, NC 27695-8205, USA (ipsen@math.ncsu.edu, http://www4.ncsu.edu/ ipsen/info.html). This research was supported in part by NSF grant DMS9714811.

1 The superscript ${ }^{*}$ denotes the conjugate transpose.

${ }^{2} I$ denotes the identity matrix.
} 
Proposition 2 (Extension of (5) in [2]). If

$$
\mathcal{P} \equiv\left(\begin{array}{cc}
A & B^{*} \\
0 & -S
\end{array}\right)
$$

then

$$
\mathcal{A P}^{-1}=\left(\begin{array}{cc}
I & 0 \\
C A^{-1} & -I
\end{array}\right)
$$

and $\mathcal{P}^{-1} \mathcal{A}$ and $\mathcal{A} \mathcal{P}^{-1}$ have the minimal polynomial $(\lambda-1)(\lambda+1)$.

The preconditioned matrix below is the same, up to permutations, as the one in $[1, \S 2.1]$.

Proposition 3. If

$$
\mathcal{P}_{1} \equiv\left(\begin{array}{cc}
I & 0 \\
C A^{-1} & -I
\end{array}\right), \quad \mathcal{P}_{2} \equiv\left(\begin{array}{cc}
A & B^{*} \\
0 & S
\end{array}\right)
$$

then

$$
\mathcal{P}_{1}^{-1} \mathcal{A} \mathcal{P}_{2}^{-1}=\left(\begin{array}{cc}
I & 0 \\
0 & -I
\end{array}\right)
$$

The preconditioned matrix is also similar to $\mathcal{P}^{-1} \mathcal{A}$ and $\mathcal{A P}^{-1}$, where

$$
\mathcal{P} \equiv\left(\begin{array}{cc}
A & B^{*} \\
C & D-2 S
\end{array}\right),
$$

which is an extension of the preconditioner in [2, $\mathrm{p} 7]$.

Remark 1. Extending the preconditioner in [2, Proposition 1] to general matrices gives

$$
\mathcal{P} \equiv\left(\begin{array}{cc}
A & \\
& -S
\end{array}\right)
$$

It can be derived from the scaled $L U$ decomposition $\mathcal{A}=\mathcal{L U D}$, where

$$
\mathcal{L} \equiv\left(\begin{array}{cc}
I & \\
C A^{-1} & I
\end{array}\right), \quad \mathcal{U} \equiv\left(\begin{array}{cc}
I & -B^{*} S^{-1} \\
-I
\end{array}\right), \quad \mathcal{D} \equiv\left(\begin{array}{cc}
A & \\
& -S
\end{array}\right)
$$

The preconditioned matrix is

$$
\mathcal{T} \equiv \mathcal{A} \mathcal{P}^{-1}=\mathcal{L U}=\left(\begin{array}{cc}
I & -B^{*} S^{-1} \\
C A^{-1} & -D S^{-1}
\end{array}\right)
$$

If $\mathcal{A}$ is of $K K T$ form with $D=0$ then

$$
\mathcal{T}^{2}-\mathcal{T}=\left(\begin{array}{cc}
-B^{*} S^{-1} C A^{-1} & 0 \\
0 & I
\end{array}\right)
$$

Since $\left(\mathcal{T}^{2}-\mathcal{T}\right)^{2}=\mathcal{T}^{2}-\mathcal{T}$, the preconditioned matrix $\mathcal{T}$ has a minimal polynomial of degree 4.

\section{REFERENCES}

[1] P. Gill, W. Murray, D. Ponceleón, and M. Saunders, Preconditioners for indefinite systems arising in optimization, SIAM J. Matrix Anal. Appl., 13 (1992), pp. 292-311.

[2] M. Murphy, G. Golub, And A. Wathen, A note on preconditioning for indefinite systems, SIAM J. Sci. Comput., 21 (2000), pp. 1969-72. 